\author{
Stefan Groh - Wolfgang Neubauer - Sirri S. Seren - Alois Eder-Hinterleitner- \\ Klaus LÖCKER
}

\title{
GEOPHYSIKALISCHE MESSUNGEN IM NORDÖSTLICHEN STADTTEIL VON FLAVIA SOLVA: INTERPRETATION UND ARCHÄOLOGISCH-HISTORISCHE AUSWERTUNG
}

\begin{abstract}
Einleitung
Im Bereich der römischen Stadt Flavia Solva (Abb. 1) sollte für die Landesausstellung 2004 eine Fläche von ca. 3 ha durch eine Georadarmessung detailliert untersucht werden. Die Messungen lagen im Nordosten des Stadtgebietes von Flavia Solva im Bereich der rechteckigen Häuserblöcke (Insulae) XXIII, XXIV, XXVI, XXVIII, XXIX, XXX, XXXV, XXVII-Süd, XXVII-Nord und XL (Abb. 2a-b) ${ }^{1}$. Eine im Rahmen einer Lehrveranstaltung der Universität Wien (W. Neubauer) und der Universität Graz (E. Hudeczek) gemeinsam mit dem Österreichischen Archäologischen Institut (St. Groh) durchgeführte erste geophysikalische Prospektion im Stadtgebiet von Flavia Solva im Jahre 1998 zeigte die gute Eignung der Fundstelle für die gewählte Methode².

Bei dieser ersten Prospektion kam die Geoelektrik in Form einer Bodenwiderstandskartierung auf den im Besitz des Landes Steiermark befindlichen Parzellen 209, 211/1, 211/2, 214 und 217 östlich des ehemaligen Museums zum Einsatz; Teile der Insulae XXIII, XXVI und XXX wurden prospektiert. Das Meßergebnis zeigte ausgesprochen starke Widerstandskontraste, die einen weiteren Einsatz des Georadars indizierten. Durch die Widerstandsmessungen konnten bereits Hinweise auf unterschiedliche Verbauungsphasen festgestellt werden, welche aber wegen der unscharfen Tiefenangaben der Widerstandskartierungen nicht näher zu interpretieren waren. Für den geplanten Einsatz des Georadars im Stadtgebiet von Flavia Solva waren damit entsprechend gute Voraussetzungen gegeben, so daß aufgrund der höheren Auflösung dieser Methode und der Möglichkeit der detaillierten dreidimensionalen Erfassung der im Boden vorhandenen Strukturen auch genauere archäologische Aussagen erwartet wurden.
\end{abstract}

\section{Geophysikalische Prospektionsmethoden}

Geophysikalische Prospektionsmethoden haben sich im letzten Jahrzehnt zu einem unverzichtbaren Hilfsmittel der Archäologie entwickelt, wobei sich besonders die Magnetik, die Bodenwiderstandsmessung und das Bodenradar in der archäologischen Anwendung bewährt haben ${ }^{3}$. Sie ermöglichen es, im Boden verborgene Strukturen durch die Messung physikalischer Eigenschaf-

\footnotetext{
${ }^{1}$ Bei dem Untersuchungsgebiet handelt es sich um die Parzellen 206/1, 206/22, 207, 208/1, 208/2, 209, 211/1, 211/2, 212/1, 212/2, 217/1, 217/2 und 248/1 der Gemeinde Leibnitz, Katastralgemeinde Wagna. Die Prospektion wurde von der Stadtgemeinde Wagna in Auftrag gegeben.

2 St. Groh - W. Neubauer - A. Eder-Hinterleitner, Ergebnisse der ersten archäologisch-geophysikalischen Prospektion im Stadtgebiet von Flavia Solva, Steiermark, ArchA 82/83, 1998/99, 27-38.

3 W. Neubauer, Magnetische Prospektion in der Archäologie, MPK 44 (2001); ders. - A. Eder-Hinterleitner P. Melichar - R. Steiner, Improvements in high resolution archaeological magnetometry, Prospezioni Archeologiche $11(2001)$.
} 


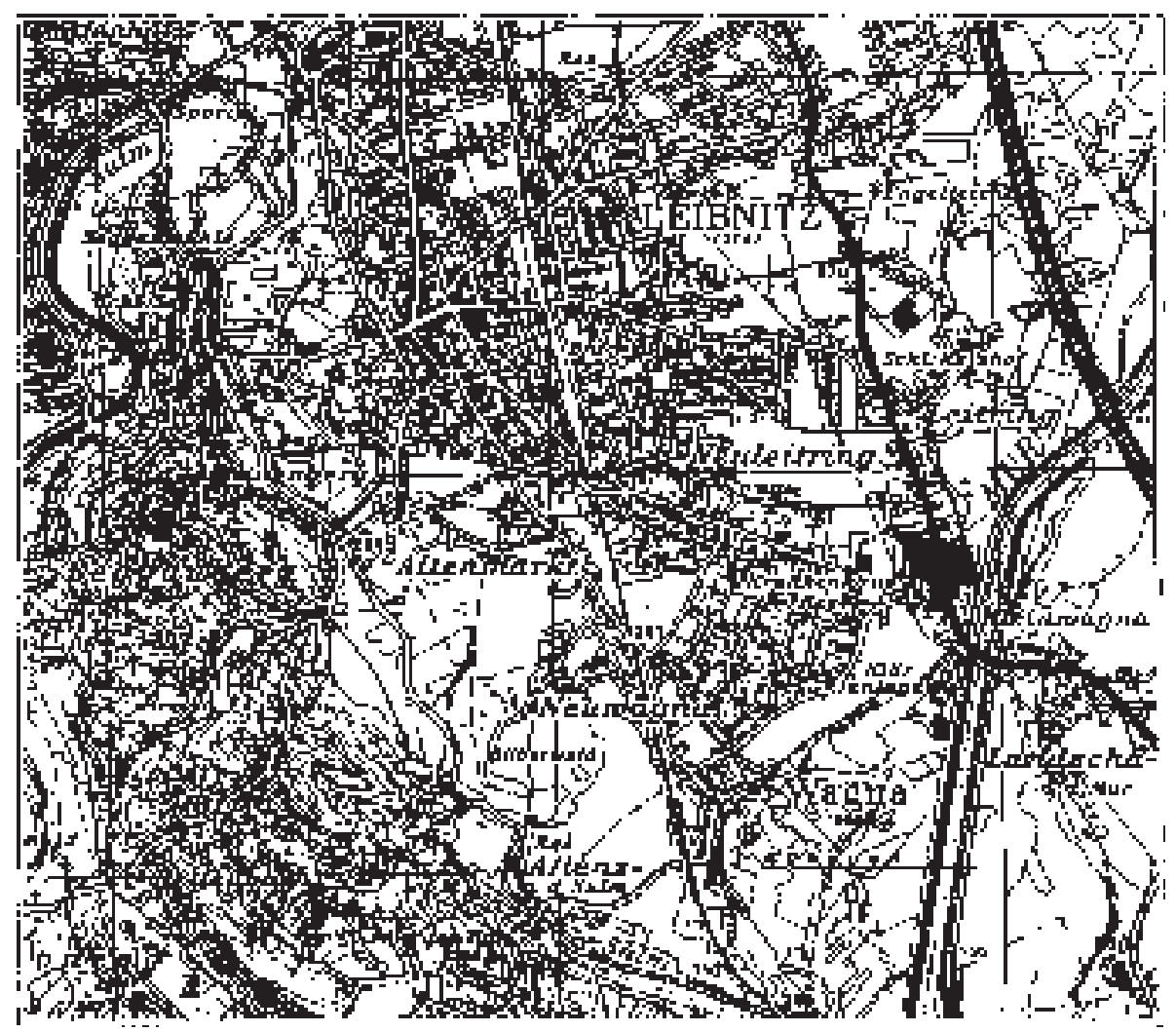

Das Gebiet der antiken Stadt Flavia Solva an der Mur

ten des Untergrundes zu erfassen und darzustellen. Für archäologische Anwendungen werden eigene Meßkonfigurationen verwendet, mit Hilfe derer eine flächige, rasterförmige Erkundung mit Meßpunktabständen von unter einem Meter in kurzer Zeit möglich ist. Für die Auswertung der großen Datenmengen werden Verfahren der digitalen Bildverarbeitung herangezogen ${ }^{4}$.

Die Leistungsfähigkeit der einzusetzenden Methoden wird vor allem durch den Kontrast der physikalischen Eigenschaften des Bodens im Vergleich zu den archäologischen Strukturen bestimmt. Eine erste Auswertung der Meßdaten erfolgt bereits vor Ort und dient der Planung des weiteren Vorgehens. Für die folgende Auswertung der großen Datenmengen werden leistungsfähige Rechner und eigens entwickelte Software verwendet. Die visualisierten Daten der einzelnen Messungen werden kombiniert und gemeinsam archäologisch interpretiert.

\section{Grundlagen des Georadars}

\section{Meßmethode}

Das Georadar ist zur Zeit die modernste und potentiell leistungsfähigste geophysikalische Methode in der archäologischen Prospektion. Es nützt eine elektromagnetische Welle als Signalträger: Ein elektromagnetischer Impuls mit der gewählten Frequenz (zwischen 100 und 900 MHz) wird mit einer Sendeantenne in den Untergrund abgestrahlt. Er breitet sich im Untergrund mit einer materialabhängigen Geschwindigkeit aus und wird von Schichten unterschiedlicher physikalischer Eigenschaften (Dielektrizitätskonstante, Leitfähigkeit) oder an den Grenzflächen einzelner

\footnotetext{
${ }^{4}$ I. Scollar - A. Tabbagh - A. Hesse - I. Herzog, Archaeological Prospecting and Remote Sensing. Topics in Remote Sensing 2 (1990); A. Clark, Seeing beneath the soil (1990).
} 
Objekte reflektiert. Das an die Oberfläche zurückkehrende elektromagnetische Signal wird von einer Empfängerantenne erfaßt und digital aufgezeichnet. Die Veränderungen der Signalform (Amplitude und Frequenz) erlauben Rückschlüsse auf die physikalischen Eigenschaften der durchstrahlten Medien wie z. B. ihre Mineralzusammensetzung, Feuchtigkeit, Porosität. Die Laufzeit des Signals ist proportional zu der Entfernung der reflektierenden Grenzfläche. Im wesentlichen wird die Ausbreitung der elektromagnetischen Wellen durch die Dielektrizitätskonstante des Mediums, die elektrische Leitfähigkeit des Mediums, die Antennenabstrahlcharakteristik und die Frequenz des Impulses beeinflußt. Der Kontrast der Dielektrizitätskonstanten zweier Medien bestimmt den Betrag der reflektierten Energie an den Schicht- und Objektgrenzen. In den obersten Boden- und Sedimentschichten treten die größten Reflexionskoeffizenten in Verbindung mit Substratwechseln, starken Inhomogenitäten, Grundwasserspiegel etc. auf. Die in den Boden abgestrahlte elektromagnetische Energie wird, abhängig vom Medium, unterschiedlich gedämpft (materialbedingter Absorbtionsverlust). Die Amplitudenabnahme des ausgesandten Energieimpulses hängt dabei im wesentlichen von der Leitfähigkeit des Mediums und dem zurückgelegten Weg ab. Die Leitfähigkeit ist die bestimmende Größe für die effektive Eindringtiefe der elektromagnetischen Wellen. Durch den Vergleich der Amplituden (Sender, Empfänger) ist es möglich, verschiedene Bereiche nach ihrer Absorbtionseigenschaft zu unterscheiden.

\section{Auswertung}

\section{Radargramm}

Die Georadardaten sind sehr umfangreich und besitzen eine hohe Informationsdichte. Die Visualisierung der Daten erfolgt meist in Form einer schwarz-weiß oder farbkodierten Darstellung der Amplituden der empfangenen Signale nach Ort und Zeit für die einzelnen Georadarsektionen und wird als Radargramm bezeichnet. Diese einzelnen Meßstreifen (der älteren analogen Meßgerätegenerationen) oder die entsprechenden digitalen Bilddarstellungen einer Sektion zeigen typische Diffraktions- und Reflexionsmuster und sind nur schwer zu interpretieren. Man kann sogar soweit gehen, zu sagen, daß der Laie wie auch der unerfahrene Archäologe aus einem Radargramm keine Interpretation ableiten kann. Aus diesem Grund werden in die Radargramme oft erklärende Linien oder Kommentare eingefügt, die ein Erfassen der erkundeten Strukturen erleichtern sollen. Diese manuelle Auswertetechnik verwendet im wesentlichen die einzelnen Georadarsektionen. Dabei wird versucht, durch optisches Vergleichen korrelierbare Diffraktions- und Reflexionsmuster zu finden und sie den archäologischen Objekten oder Strukturen zuzuweisen. Das Ergebnis dieser subjektiven Interpretation ist immer von der Erfahrung und dem Wissen des Geophysikers abhängig und kaum reproduzierbar. Die so in den Georadarsektionen gefundenen Anomalien bilden die Grundlage für die handgezeichneten Anomalienflächenpläne, die meist die dreidimensional vorhandene Information in kompilierter Form auf die Oberfläche projiziert darstellen. Tiefenangaben zu den Anomalien erfolgen, wenn überhaupt, nur punktuell.

Eine solche reduzierte zweidimensionale Auswertung mit Erstellung eines kompilativen Interpretationsplans nützt nur einen geringen Teil der in den Daten vorhandenen Informationen. Mit dieser Auswertetechnik können die schwachen Störungen und die Tiefenausdehnung archäologischer Strukturen kaum oder selten ausgewiesen werden. Dies führte meist zu enttäuschenden Ergebnissen für den Archäologen und damit zu einem negativen archäologischen Feedback gegenüber dem Georadar.

Um das hohe archäologische Potential des Georadars zu nutzen, ist der Einsatz neuer Auswertungsmethoden notwendig, damit reproduzierbare und objektive Ergebnisse erzielt werden können ${ }^{5}$. Wesentliche Vorarbeiten im archäologischen Anwendungsfeld von

\footnotetext{
5 D. Goodman, Ground penetrating radar simulation in engineering and archaeology, Geophysics 59, 2, 1994, 224-232.
} 


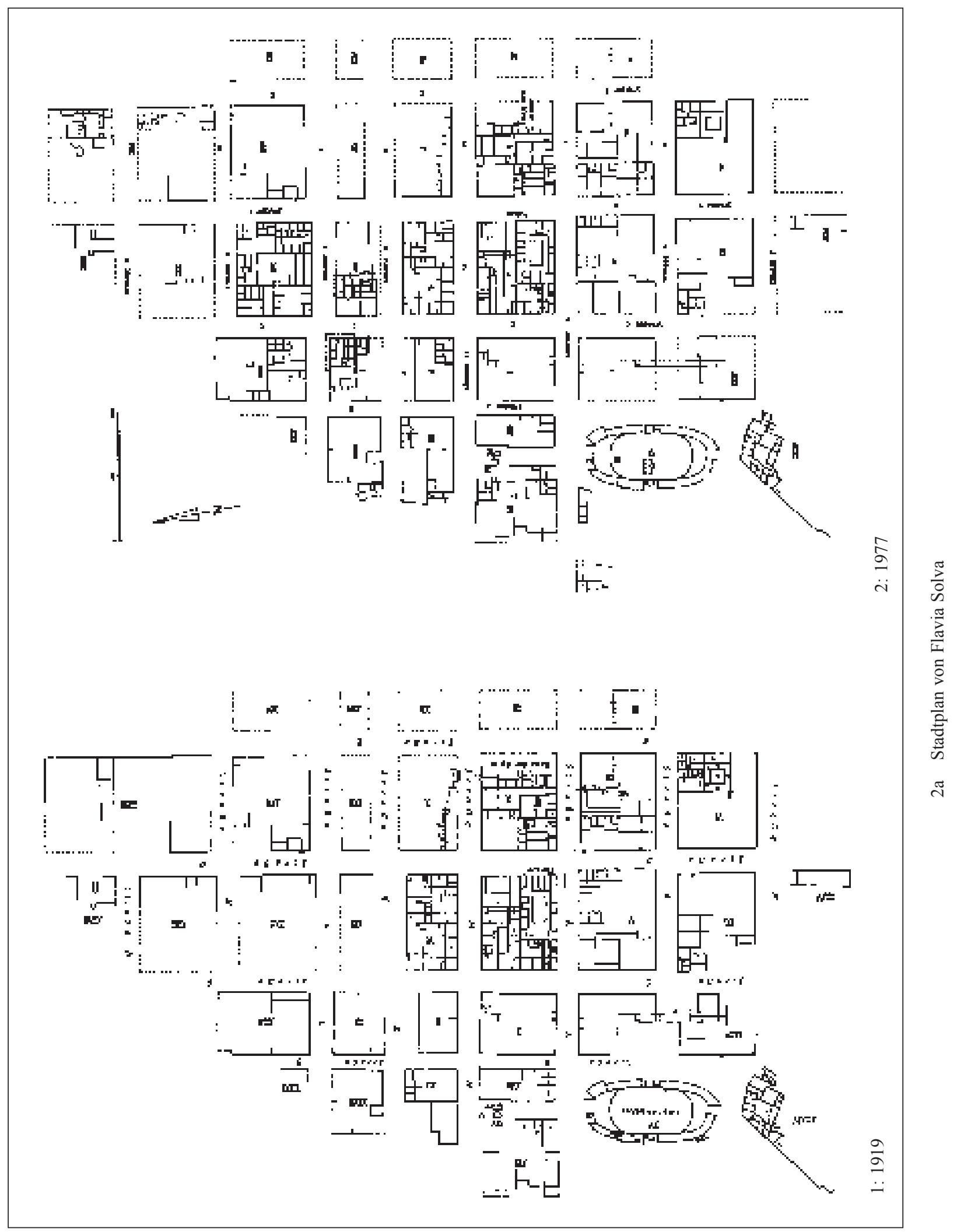




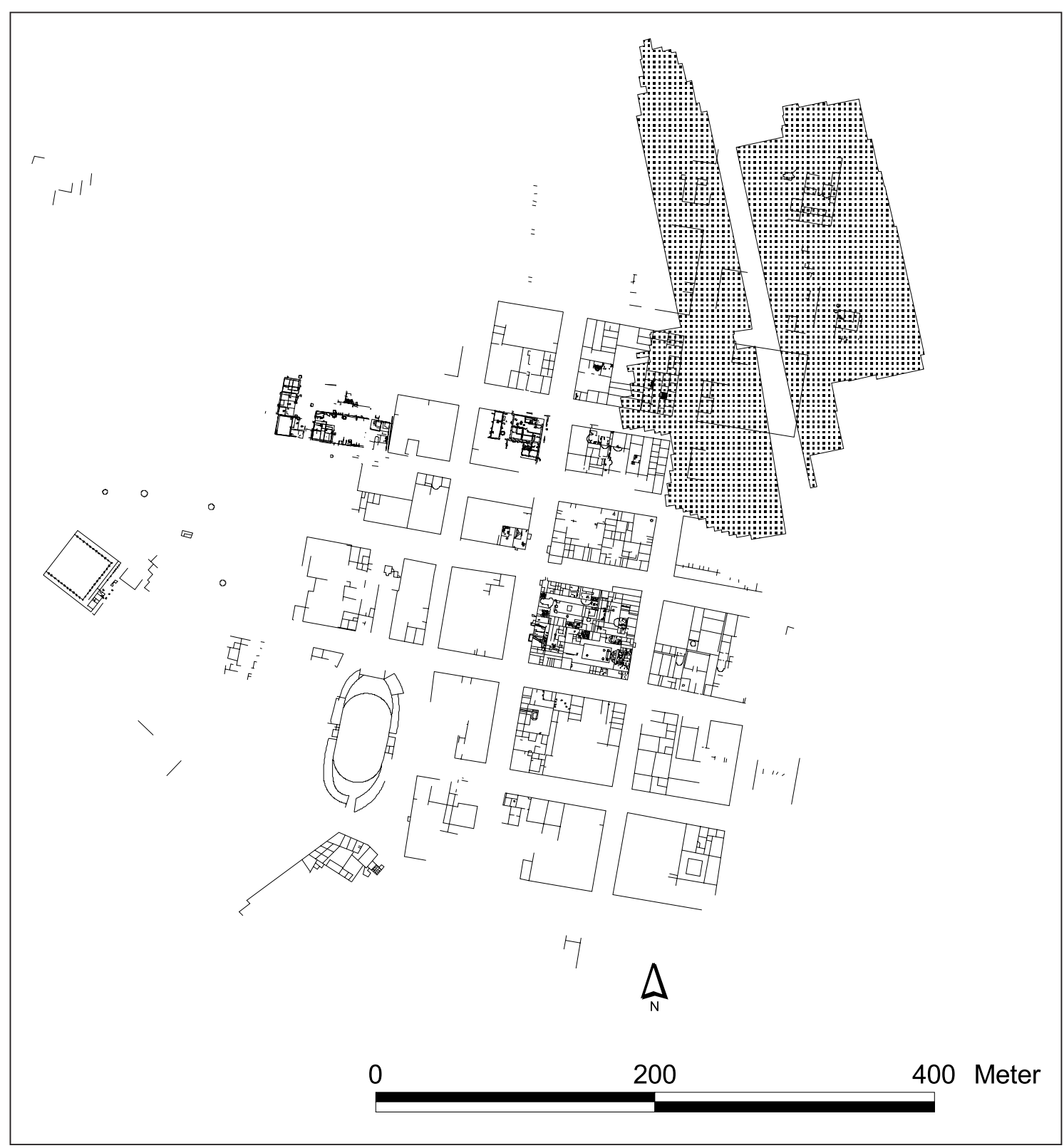

2b Stadtplan von Flavia Solva 2001, grau gerastert die Meßflächen 2000

S. Lorra ${ }^{6}$ propagierten die konsequente rechnergestützte, flächenhafte Visualisierung der Daten in Form von Amplitudenflächenplänen in diskreten Tiefen. Archeo Prospections ${ }^{\circledR}$ erweiterte diesen Ansatz konsequenterweise auf einen dreidimensionalen Datenblock mit der Möglichkeit beliebiger drei- oder zweidimensionaler Visualisierungen als Grundlage für die weitere archäologische Interpretation ${ }^{7}$.

${ }^{6}$ S. Lorra, Geophysikalische Prospektion und Modellierung archäologischer Fundplätze in Schleswig-Holstein, Universitätsforschungen zur prähistorischen Archäologie 36 (1996).

7 W. Neubauer - A. Eder-Hinterleitner - S. Seren - M. Doneus - P. Melichar, Kombination archäologisch-geophysikalischer Prospektionsmethoden am Beispiel der römischen Zivilstadt Carnuntum, ArchA 82/83, 1998/99, 1-26. 
Geschwindigkeitsanalyse

Eine Analyse der Ausbreitungsgeschwindigkeit der Radarwellen kann durch die Auswertung der CMP(common-mid-point)-Messungen oder durch Anpassung der Diffraktionshyperbeln bekannter Objekte durchgeführt werden.

\section{Amplitudenverteilung}

Die Verteilung der Amplituden der Reflexionen in einem bestimmten Zeitbereich wird als Zeitscheibe (time-slice) bezeichnet. Es wird dabei der Absolutbetrag der Amplitude für die Berechnung der Zeitscheiben verwendet, eine Methode, die sich für archäologische Aufgabenstellungen sehr gut bewährt hat. Der Absolutbetrag der Amplitude in den jeweiligen Tiefenbereich, der sich durch die bekannte Ausbreitungsgeschwindigkeit bestimmen läßt, wird durch Aufsummieren der Amplitudenbeträge über ein Zeitintervall berechnet. Die Amplitudenwerte sind ein Maß für die Reflektivität des Untergrundes in einem bestimmten Tiefenintervall. Während hohe Amplitudenwerte relativ zur Umgebung auf erhöhte Reflektivität durch Strukturen und Objekte wie beispielsweise Mauern deuten, repräsentieren niedrige Amplitudenwerte die stark dämpfenden, humosen oder lehmigen Schichten. Alle Amplitudenflächenpläne ergeben zusammen ein dreidimensionales Bild des untersuchten Tiefenbereiches. Sie bilden auch die Grundlage für die archäologische Interpretation und eine spätere Einbindung in ein Geographisches Informationssystem (GIS).

\section{Datenanalyse und Datenvisualisierung}

Zur Analyse wird der dreidimensionale Datenblock in das wissenschaftliche Visualisierungsprogramm AVS (Advanced Visualisation Systems) eingelesen. Weiters werden zweidimensionale Bilder durch Schnitte des Datenblocks mit horizontalen und vertikalen Ebenen berechnet und mit kommerziellen Softwareprodukten visualisiert. Es können beliebige Zeitbereiche zu einem Bild zusammengefaßt werden, um die archäologische Interpretation zu erleichtern.

Durch Animation dieser zweidimensionalen Visualisierungen in x-, y- oder z-Richtung sind Filmsequenzen herstellbar, welche sowohl in der Ablaufgeschwindigkeit als auch in der Ablaufrichtung gesteuert werden können und somit völlig neue Eindrücke der Amplitudenverteilungen entstehen lassen. Ein virtuelles Abtragen des Meßblockes in horizontalen oder vertikalen Schichten wird veranschaulicht. Dadurch wird eine explorative Datenanalyse möglich, die zu schrittweisem mentalem Erfassen der komplexen archäologischen Strukturen führt. So sind beispielsweise schräg nach unten verlaufende Strukturen wie Treppen durch eine Animation horizontaler Schichten sofort erkennbar.

\section{Georeferenzierung und Kombination}

Zur Interpretation werden die ausgesuchten horizontalen Schnitte durch den dreidimensionalen Datenblock der Amplitudenverteilung georeferenziert und mit allen bestehenden Informationen (weiteren Prospektionsdaten, Katasterplänen, Grabungsplänen, Interpretationen etc.) im GIS (Arc View) kombiniert. Durch Übereinanderlegen von Visualisierungen dieser verschiedenen Informationen ist eine beliebige Kombination sehr einfach und schnell durchführbar und somit eine interaktive Überprüfung von Korrelationen möglich.

\section{Georadar}

Das Programm APRADAR, welches von Archeo Prospections ${ }^{\circledR}$ für schnelle Auswertung von Georadardaten in der archäologischen Prospektion für den PC entwickelt worden ist, bildet die Basis der hier angewandten Auswertungsmethode. Es erlaubt die gemeinsame Verarbeitung und die dreidimensionale Auswertung von allen Georadarprofilen aller Meßflächen entsprechend ihrer Lagekoordinaten.

Die wesentlichen Schritte dieser Auswertung sind folgende: Die PulseEKKO ${ }^{\circledR}$-Meßdaten werden eingelesen und für die Weiterverarbeitung umformatiert. Die Akquisition- und Geometrieparameter werden editiert und korrigiert. An jeder Meßspur wird die Startzeit bestimmt und 


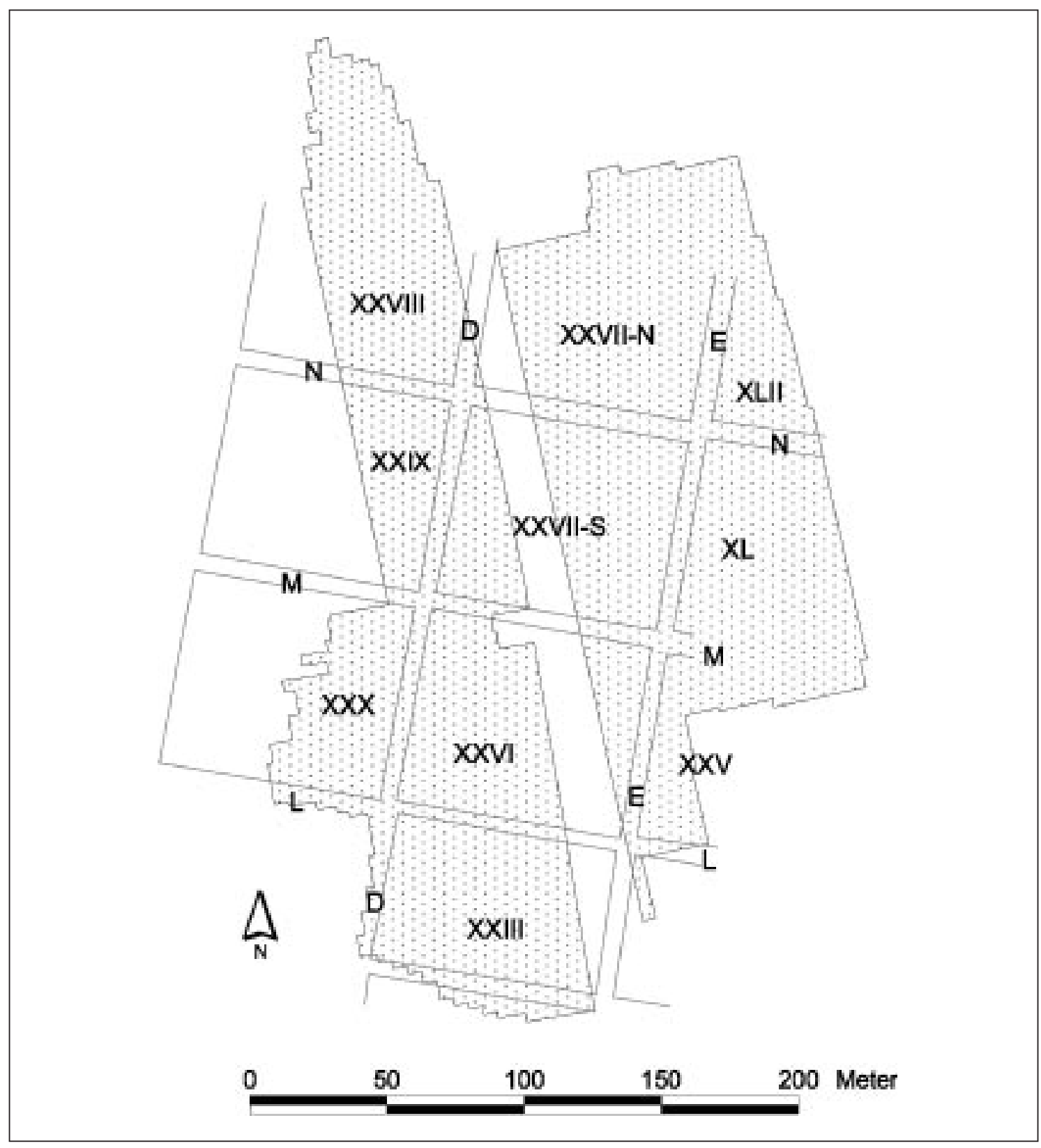

3 Flavia Solva. Numerierung und Bezeichnung der durch die Georadarmessungen erfaßten Insulae und Straßen

korrigiert. Amplitudenflächenpläne (Zeitscheiben) werden berechnet. Die Amplituden der Zeitscheiben werden durch Berechnen der Quadratwurzel nichtlinear abgebildet. Dadurch werden kleinere Amplituden im Vergleich zu sehr großen verstärkt und Details können in der Visualisierung besser wahrgenommen werden. Der Energieverlust der Zeitscheiben mit der Tiefe wird durch multiplikativen Angleich aller Zeitscheiben an einen gemeinsamen Mittelwert beseitigt. Alle Zeitscheiben können dadurch im selben Bereich visualisiert werden. Zur Beseitigung streifenartiger Muster entlang der Meßlinienrichtung, welche durch sehr kleine Startzeitkorrekturfehler bedingte generelle Amplitudenverschiebungen oder durch zwischenzeitliches Abschalten des Meßgerätes entstehen können, werden die Amplitudenwerte entlang der Meßprofile durch Addition eines konstanten Wertes an die benachbarten Profile oder an den gesamten Mittelwert der Zeitscheibe angeglichen. Filterungen sowohl entlang der Meßprofilrichtung als auch unter 


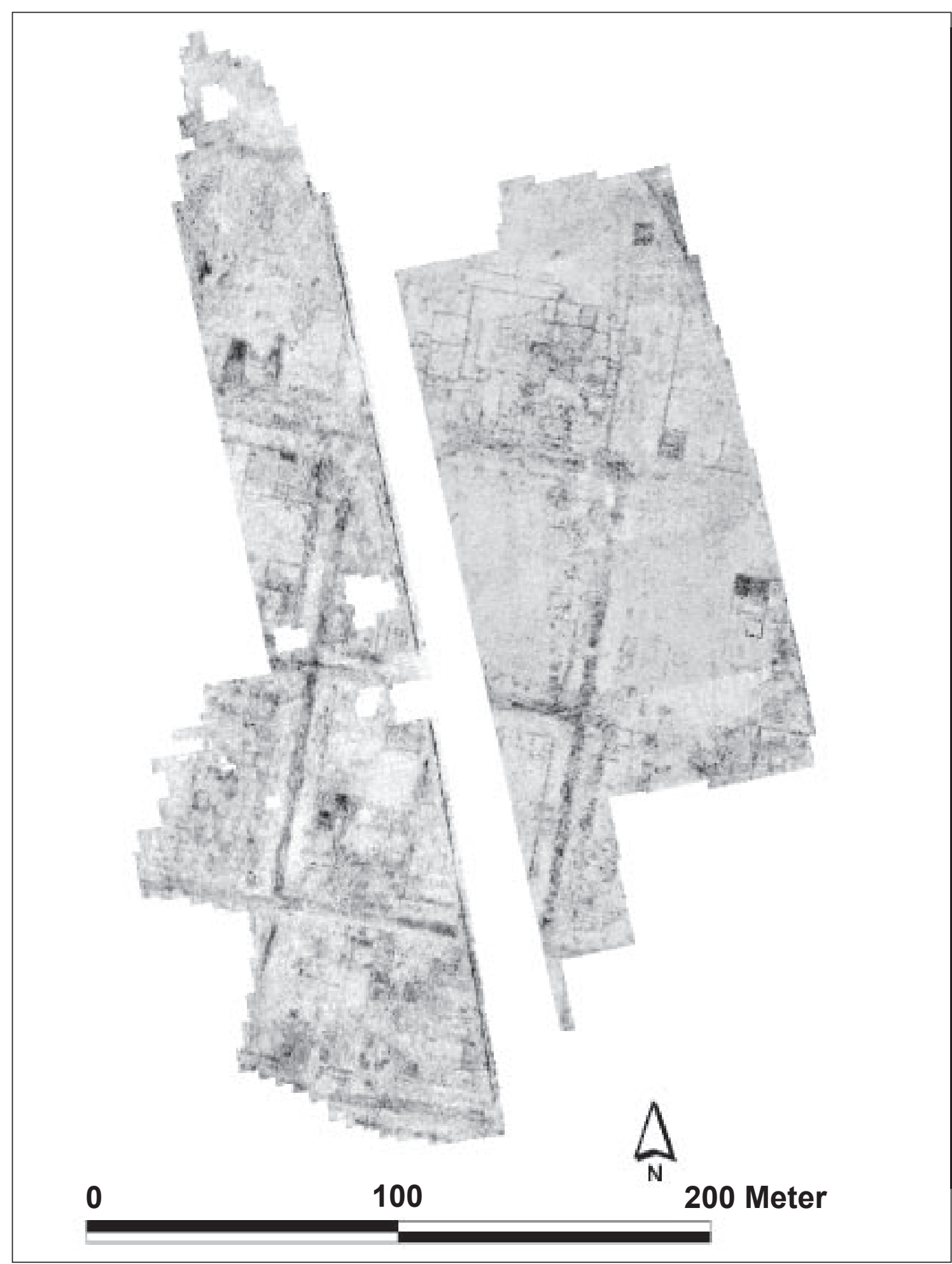

4 Georadarmessungen im Nordostteil von Flavia Solva bei einer Eindringtiefe von $0,8 \mathrm{~m}$

Einbeziehung benachbarter Meßprofile werden zur Glättung der Amplitudenverteilung eingesetzt. Damit die Amplitudenverteilung jedoch nicht unscharf wird, müssen diese sehr sorgfältig ausgewählt werden. Es wird aus allen berechneten Amplitudenflächenplänen ein dreidimensionaler Datenblock erzeugt. Aus diesem werden wiederum horizontale und vertikale zweidimensionale Schnitte berechnet, die mit den kommerziellen Softwareprodukten visualisiert werden können. Auch beliebige Zeitscheiben können zu einem Bild zusammengefaßt werden, um die 
archäologische Interpretation zu erleichtern. Für die archäologischen Interpretation werden die Amplitudenflächenpläne im GIS georeferenziert.

Dr. Wolfgang Neubauer

Dr. Sirri S. Seren

DI Alois Eder-Hinterleitner

Mag. Klaus Löcker

Archeo Prospections ${ }^{\mathfrak{B}}$, Zentralanstalt für Meteorologie und Geodynamik, Hohe Warte 38, A-1191 Wien

E-Mail: archeo@zamg.ac.at

\section{Die archäologisch-historische Interpretation der Georadardaten von Flavia Solva}

Im Zuge der Auswertung der geophysikalischen Prospektion wurde am Österreichischen Archäologischen Institut ein neuer digitaler Plan von Flavia Solva generiert, der alle publizierten Übersichts- und Detailpläne von Flavia Solva und den Gräberfeldern enthält ${ }^{8}$. Als Grundlage für diesen Stadtplan wurden folgende Quellen berücksichtigt: die Stadt- und Gräberfeldpläne von W. Schmid ${ }^{9}$, W. Modrijan ${ }^{10}$, E. Staudinger ${ }^{11}$, E. Hudeczek ${ }^{12}$, G. Fuchs ${ }^{13}$, G. Fuchs u. a. ${ }^{14}$ und St. Groh ${ }^{15}$ sowie Detailpläne einzelner Insulae, die hier bei der Besprechung der jeweiligen Baukomplexe zitiert werden (Abb. 2a. b). Der bestehende Stadtplan von Flavia Solva wurde aufgrund von Paßpunkten, die aus den geophysikalischen Meßergebnissen ermittelt wurden, durch entsprechende Transformationen präzisiert.

Von den Georadarmessungen wurde der Bereich der Insulae XXIII, XXIV, XXVI, XXVIII, XXIX, XXX, XXXV, XXVII-Süd, XXVII-Nord und XL bzw. der Nord-Süd verlaufenden Straßen D und E und der Ost-West verlaufenden Straßen K, L, M und N erfaßt (Abb. 3. 4). Die Begrenzung der Meßfläche in Richtung Osten wird von einem steilen Abbruch zum Flußtal der Mur gebildet. Die Meßfläche gliedert sich in zwei getrennt gemessene Bereiche westlich und östlich der Bundesstraße B67. Das Ergebnis der Georadarmessung kann aufgrund der starken Kontraste als ausgesprochen gut bezeichnet werden (Abb. 5. 6).

Die Eindringtiefe der Georadarmessung im Gebiet westlich der Bundesstraße, wo mit einer 450-MHz-Antenne gemessen wurde, beträgt 2,5 m. Damit wurde in allen Bereichen dieser Meßfläche bis unter die tiefsten Fundamente der archäologischen Strukturen gemessen. Die Eindringtiefe im Gebiet östlich der Bundesstraße, wo mit einer 900-MHz-Antenne gemessen wurde, beträgt hingegen nur 1,4 m. Dadurch wurden einzelne, tief reichende Fundamente nicht mehr vollständig erfaßt. Für die folgende archäologische Interpretation ist die Eindringtiefe jedoch als ausreichend zu betrachten.

Für die endgültige archäologische Interpretation wurden drei Amplitudenflächenpläne für die Tiefenbereiche 0,5-1,0 m, 1,0-1,5 $\mathrm{m}$ und 1,5-2,5 $\mathrm{m}$ erstellt. Bei der Interpretation wurden die erkannten Befunde getrennt nach Straßen, sicheren Mauerverläufen, möglichen Mauerver-

\footnotetext{
${ }^{8}$ Für Anregungen und die Diskussionsbereitschaft danken wir E. Hudeczek (Steiermärkisches Landesmuseum Joanneum), für Digitalisierungsarbeiten und Literaturrecherche I. Benda-Weber und J. Wagner (ÖAI Wien).

9 W. Schmid, Flavia Solva bei Leibnitz in Steiermark, ÖJh 19/20, 1919, Beibl. 141 ff. Abb. 61. 62.

10 W. Modrijan, 1900 Jahre Flavia Solva - Flavia Solva und seine Erforschung, SchStKlSchr 11 (1971) Faltplan.

1 Privatarchiv E. Staudinger.

12 E. Hudeczek, Flavia Solva, in: ANRW II 6 (1977) 448 Abb. 5.

13 G. Fuchs, Die römerzeitlichen Gräberfelder von Flavia Solva (ungedr. Diss. Graz 1980) Beilagen.

${ }^{14}$ G. Fuchs - St. Groh - I. Kainz - G. Pachler, Archäologische Landesaufnahme und digitaler Fundkataster für Steiermark, Jahresbericht 1989 (1990) 42 ff. Abb. 5, 1.

15 St. Groh, Die Insula XLI von Flavia Solva. Ergebnisse der Grabungen 1959 und 1989 bis 1992, SoSchrÖAI 28 (1996) Plan 11-14.
} 


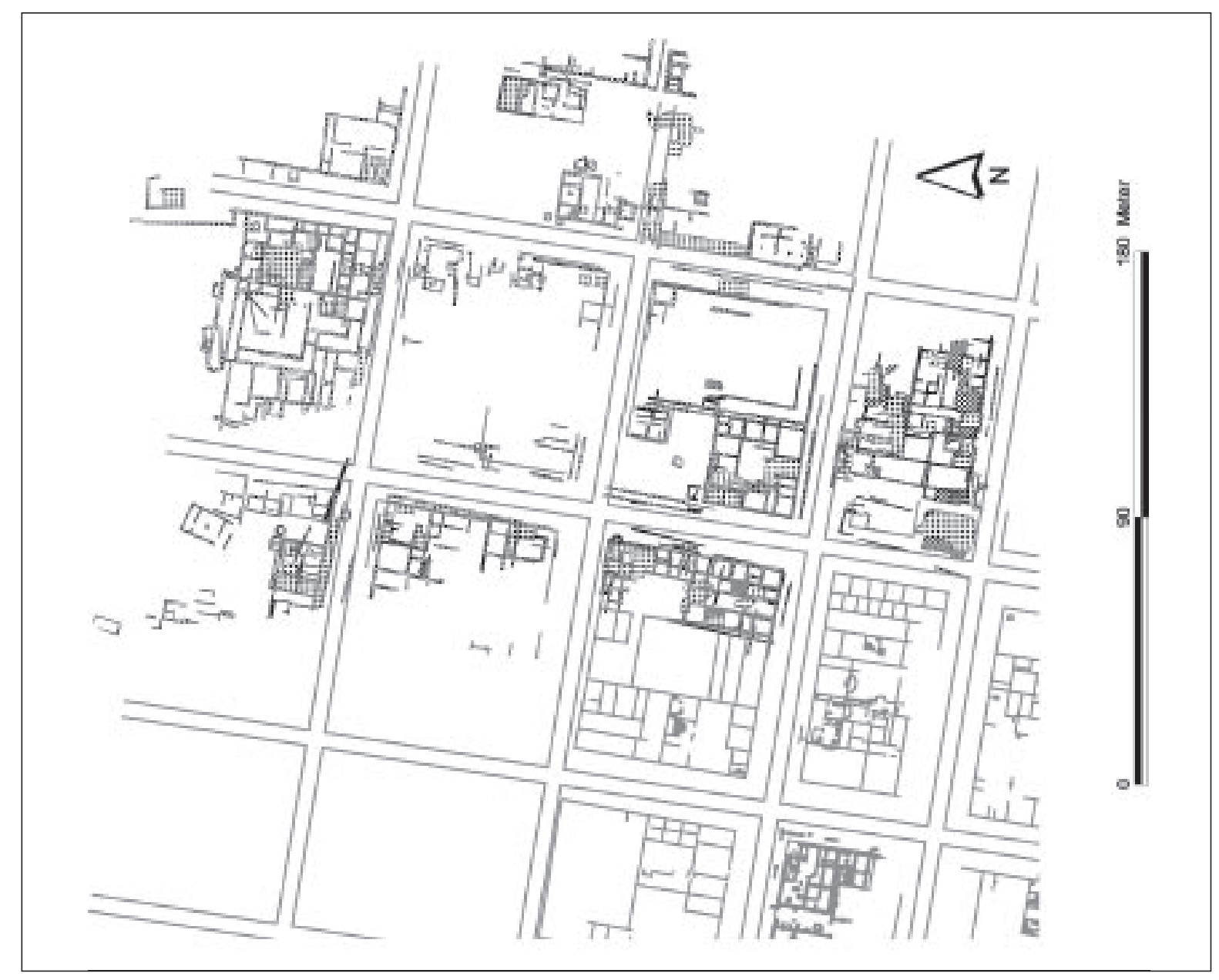

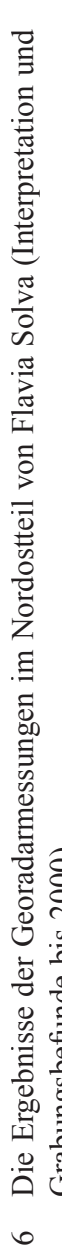

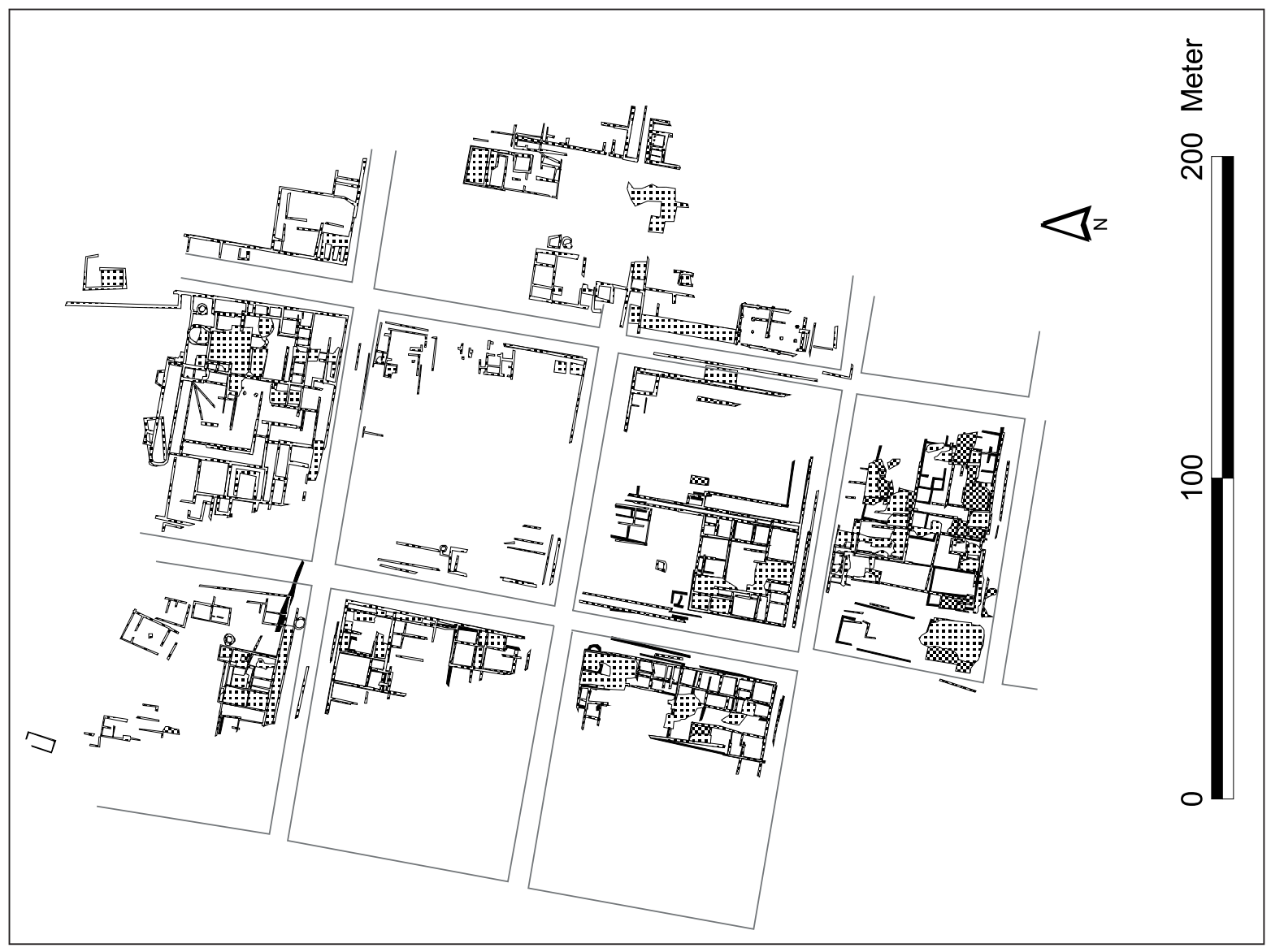

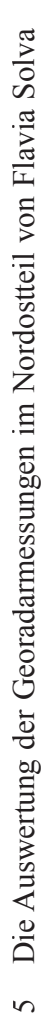


läufen und Estrichen oder Steinlagen innerhalb von Gebäuden umgezeichnet. Auf Basis der drei Amplitudenflächenpläne wurden drei archäologische Interpretationspläne erstellt.

\author{
Die Straßen D, E, K, L, M und $\mathrm{N}^{16}$
}

Sehr deutlich läßt sich in den Amplitudenflächenplänen der rechtwinkelige Straßenraster von Flavia Solva erkennen (Abb. 4). Dem Stadtplan römischer Städte liegt ein Straßenraster zugrunde, für dessen Anlage mehrere Faktoren ausschlaggebend waren ${ }^{17}$ und dessen Vermessung mit Hilfe der Groma erfolgte, wobei am Beginn die zwei Hauptachsen der Siedlung - cardo und decumanus - definiert wurden. In Flavia Solva wurden diese Hauptachsen (Straßen D und K) durch die Dimension der sie flankierenden Gebäudekomplexe - Insulae - deutlich akzentuiert ${ }^{18}$.

Die Straßen von Flavia Solva setzten sich aus dem eigentlichen, geschotterten und mehrmals erhöhten Straßenkörpern und den zumeist von Laubengängen gesäumten Randbereichen zusammen (Abb. 3). Die östlich der Straße D in einem Abstand von $88 \mathrm{~m}$ (gemessen jeweils von der Mitte der Straßen) parallel verlaufende Straße E zeigt eine Breite der Schotterung von 5,5-6,5 m. Sie wurde auf einer Länge von $217 \mathrm{~m}$ erfaßt. Im nördlichen Bereich beträgt die gesamte Breite der Straße 15 m, im Süden zwischen den Insulae XXVI und XXV verengt sich die Straße auf eine Breite von 12,7 m; nördlich der Insula XXVII-Nord kann keine weiterlaufende Schotterung festgestellt werden. Die Straßen in der durch die Georadarmessung erfaßten Meßfläche zeigen unterschiedliche Breiten und werden im Folgenden genauer beschrieben. Der prospektierte Verlauf der West-Ost führenden Straßen wird jeweils durch die Bundesstraße B67 unterbrochen.

Die Breite der Straßenschotterungen dürfte über die Jahrhunderte kaum variiert haben, was die Untersuchungen von W. Schmid vor 1912 in der zwischen den Insulae IX und VIII gelegenen Straße A bezeugten. Anhand seiner Profilbeschreibung ließen sich vier Straßenniveaus bei 0,22, $0,81,1,01$ und 1,23 m unter der Geländeoberkante unterscheiden ${ }^{19}$.

\title{
Straße D
}

Die Straße D, welche die Stadt von Norden nach Süden durchläuft, weist eine unterschiedlich breite Schotterung von 4,5-6,5 $\mathrm{m}$ auf. Sie wurde auf einer Länge von $233 \mathrm{~m}$ durch die Messung erfaßt. Ihre gesamte Breite beträgt $16 \mathrm{~m}$, wobei bei den Insulae XXX, XXVI, XXIX und XXVIII Laubengänge (Portiken) mit einer Breite von 2,5-2,7 m nachzuweisen sind. In den Bereichen mit beidseitig die Straße begleitenden Portiken verengt sich die Straße auf eine gesamte Breite von $11 \mathrm{~m}$. Es handelt sich bei der Straße D um die breiteste, durch die Messung erfaßte Nord-Süd verlaufende Straße im Stadtgebiet von Flavia Solva.

\section{Straße N}

Die im Norden gelegene West-Ost führende Straße N wurde von der Messung auf einer Länge von $180 \mathrm{~m}$ erfaßt. Sie weist eine im Durchschnitt $6,0 \mathrm{~m}$ breite Schotterung auf. Im Bereich der Insulae XXXVIII und XXIX war die Straße 16 m breit; hier lassen sich auch Portiken nachweisen, die den freien Straßenraum auf $12 \mathrm{~m}$ einengten. Die Straße N scheint die nördlichste, West-Ost verlaufende Straße des Stadtgebietes von Flavia Solva gewesen zu sein.

\footnotetext{
16 Die Benennung der Straßen richtet sich nach dem Stadtplan von E. Hudeczek (Anm. 12) 448 Abb. 5.

17 Th. Lorenz, Römische Städte (1987) 41 ff.

$18 \mathrm{Zu}$ Straßenraster und Morphologie von Flavia Solva s. Groh (Anm. 15) $167 \mathrm{ff}$.

19 W. Schmid, Forschungen in Flavia Solva bei Leibnitz, ÖJh 15, 1912, 41 Anm. 1.
} 

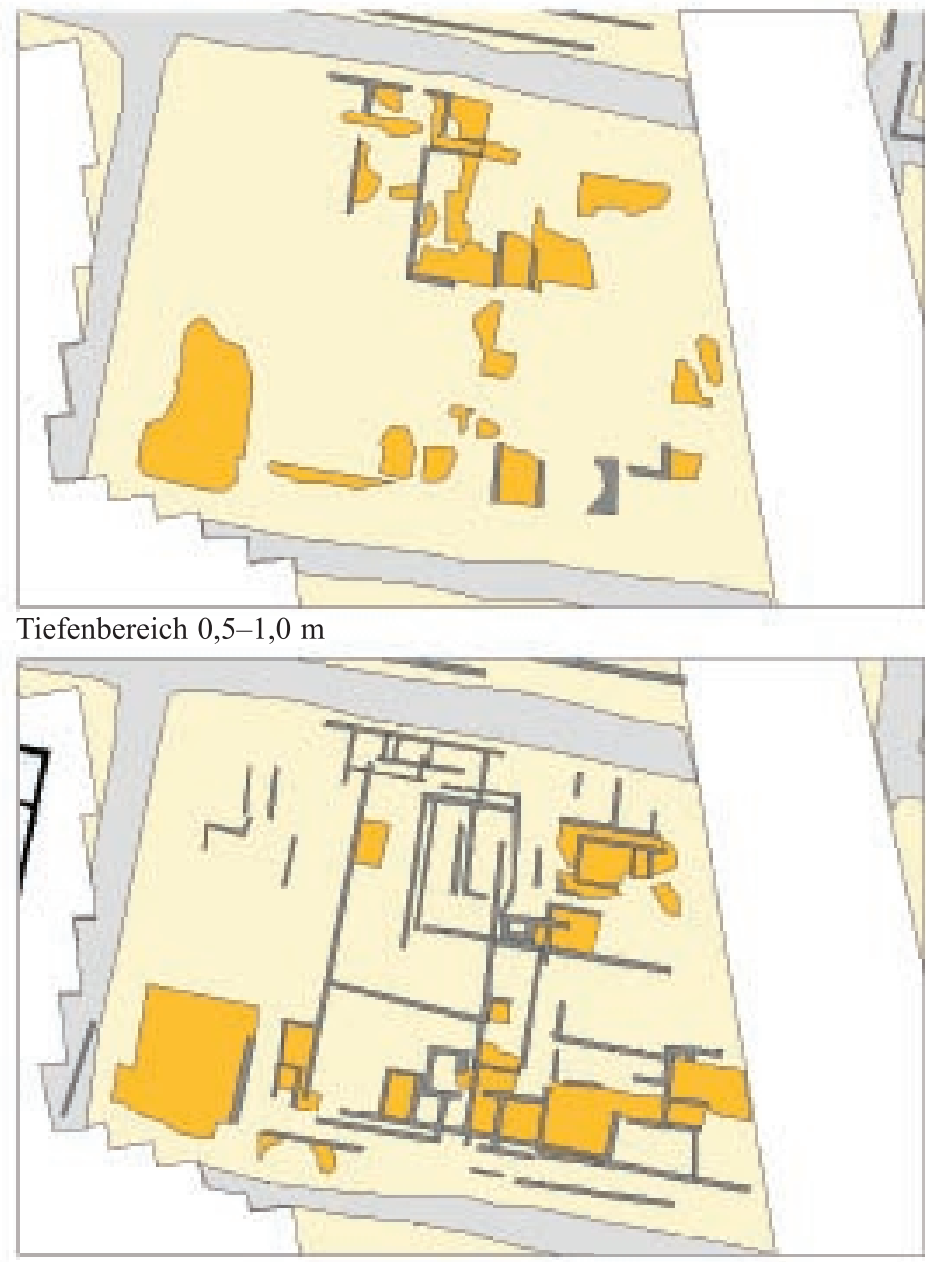

Tiefenbereich $1,0-1,5 \mathrm{~m}$

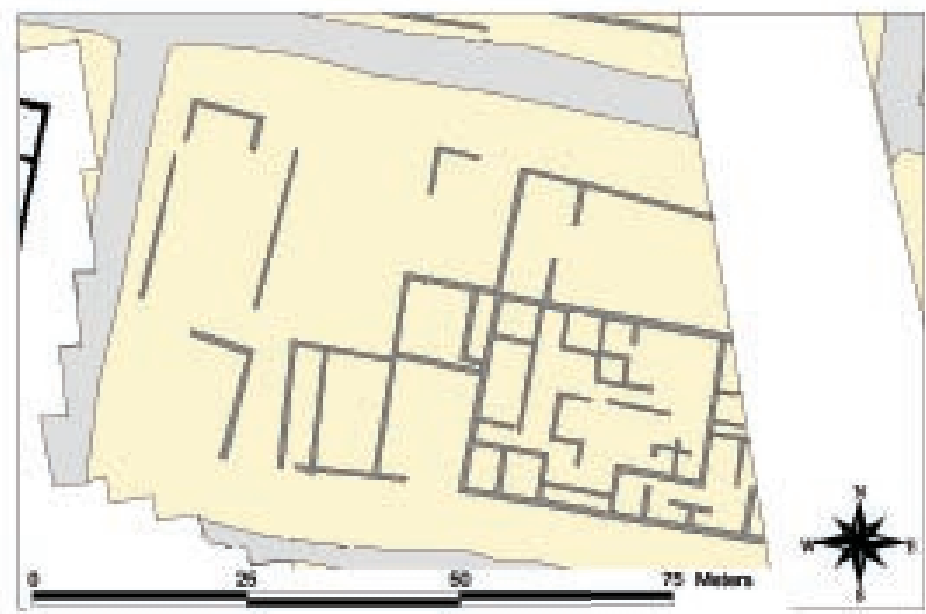

Tiefenbereich $1,5-2,5 \mathrm{~m}$

Legende

Estrich

Mauer

Straße

Prospektierte Fläche

7 Flavia Solva. Interpretation der Georadarmessungen in der Insula XXIII

\section{Straße $M$}

Die zu der Straße $\mathrm{N}$ in einem Abstand von $80 \mathrm{~m}$ parallel verlaufende Straße $M$ weist eine Schotterung mit einer mittleren Breite von 5,5-6,5 m auf. Ihre Gesamtbreite beträgt $15 \mathrm{~m}$ und verengt sich im Bereich mit beidseitigen Portiken auf $11 \mathrm{~m}$. Diese Straße scheint bei den Insulae XXV und XL abzubrechen. Die östlich nachweisbaren Gebäudestrukturen respektieren zwar noch die Straßenachse, ein weiterer Verlauf der Straße kann aber nicht nachgewiesen werden.

\section{Straße L}

Die in einem Abstand von $75 \mathrm{~m}$ zur Straße M parallel verlaufende Straße L weist eine Breite der Schotterung von 5,0-6,0 $\mathrm{m}$ auf und wurde bei der Messung auf einer Länge von $156 \mathrm{~m}$ erfaßt. Die gesamte Breite beträgt $11 \mathrm{~m}$, wobei im Bereich der Insula XXVI eine Portikus festgestellt werden kann, welche die Straße auf eine Breite von 7,7 m verengt.

\section{Straße $K$}

Die in einem Abstand von $60 \mathrm{~m}$ südlich der Straße L parallel verlaufende Straße K konnte auf einer Länge von nur $73 \mathrm{~m}$ durch die Messung erfaßt werden. Die nachweisbare Schotterung besitzt eine Breite von 4,5-6,0 m, wobei eine Straßenbreite zwischen beidseitigen Portiken von 10,0 m anzunehmen ist. Es handelt sich somit, wie bei Straße D, ebenfalls um eine breite Straße. 
Die prospektierten Gebäudekomplexe im Nordostteil der Stadt (Abb. 3-6)

\author{
Insula XXIII
}

Forschungsgeschichte

In der Insula XXIII fanden unter W. Schmid nicht näher spezifizierbare Grabungen statt, auf Schmids Stadtplan von 1919 wurden Teile einer östlichen Außenmauer eines Gebäudekomplexes kartiert (Abb. 2a ${ }^{20} .1998$ erfolgte die erwähnte erste geophysikalische Prospektion von Flavia Solva mit Widerstandsmessungen auf Flächen der Insulae XXIII, XXVI und XXX ${ }^{21}$. Die Interpretation der Meßergebnisse bezeugte eine mehrphasige Verbauung dieses Gebäudekomplexes mit einem dichter bebauten Ostteil und einer Vielzahl gut ausgestatteter Räume mit Mörtelböden. Erstmals konnte auch die Größe der Insula mit ca. 72 zu 40 m näher bestimmt werden, sie entspricht somit in ihren Dimensionen der benachbarten Insula XXII. 1999 fanden auf demselben Areal durch Joanneum Research Forschungsgesellschaft Messungen mit Georadar statt ${ }^{22}$. Die Ergebnisse dieser bezeugen einen die Gebäude säumenden Laubengang im Norden, Osten und Süden sowie einen ebenfalls intensiver bebauten Ostteil der Fläche. Im Detail können die publizierten Ergebnisse jedoch nicht mit denjenigen der Widerstandsmessungen verglichen werden, weshalb diese Insula im Jahr 2000 erneut gemessen wurde.

\title{
Interpretation der geophysikalischen Prospektionsergebnisse
}

Vor Beginn der Prospektionsmessungen waren von dieser Insula weder das Ausmaß noch eine Bebauung bekannt. Die Insula XXIII ist ein schmaler, ca. $48 \times 75 \mathrm{~m}$ messender Gebäudekomplex, der in seinem Ostteil von der Bundesstraße B67 überlagert wird. Die Insula konnte durch die Messung auf einer Fläche von ca. $3400 \mathrm{~m}^{2}$ untersucht werden (Abb. 7). Die in den Radargrammen erkennbaren Innenstrukturen zeigen deutlich eine mehrphasige Verbauung. Innerhalb der Räume können Estrichreste bzw. Schutt festgestellt werden. Es kann eine Parzellierung der Insula nachgewiesen werden, und zwar eine Halbierung entlang der Nord-Süd-Achse (Abb. 35). Die Bebauung des Westteils unterschied sich grundlegend von der des Ostteils: Die westliche Hälfte der Insula scheint in vier streifenförmige Parzellen mit Breiten von 7,8-10,0 m unterteilt gewesen zu sein. Das westlichste Drittel der Insula zeigt eine geringe Verbauung. Die tiefsten Fundamentunterkanten reichen bis 1,8 m unter Geländeoberkante, sie gehören zu zwei streifenförmigen Parzellen mit Häusern im mittleren Westteil der Insula, die eine Innenteilung durch längsrechteckige Korridore aufwiesen. Diese Bebauung erinnert an Tabernae, wie sie z. B. im Südostteil der Zivilstadt von Enns/Lauriacum zu finden $\operatorname{sind}^{23}$. Das östliche Haus war ungefähr $24 \times 10 \mathrm{~m}$ groß, das westliche mit $12 \times 10 \mathrm{~m}$ annähernd quadratisch. Innerhalb der streifenförmigen Parzellen gab es (jüngere?) seichter fundamentierte Einbauten bzw. in einer zweiten Phase eine über die gesamte Insulabreite durchgehende streifenförmige Bebauung.

Das östlichste der längsrechteckigen Gebäude $(16 \times 6,5 \mathrm{~m})$ weist in der Tiefe von $0,8 \mathrm{~m}$ unter Geländeoberkante einen interessanten Grundriß auf: Im Abstand von $0,9 \mathrm{~m}$ verlief parallel zu der westlichen und östlichen Außenmauer je eine Innenmauer, die den hallenartigen Raum in einen ca. $2 \mathrm{~m}$ breiten Mittelgang und die beiden ca. $0,9 \mathrm{~m}$ breiten Seitenkorridore unterteilte. Im Süden scheint es einen Abschluß des mittleren Ganges gegeben zu haben, bzw. war den drei Korridoren ein ca. $2 \mathrm{~m}$ tiefer Raum vorgelagert (Abb. 8). Dieser Grundriß erinnert, mit aller

\footnotetext{
20 Schmid (Anm. 9) 141 Abb. 61.

21 Groh u. a. (Anm. 2) 29. 32-35.

22 R. Fruhwirth - G. Fuchs - R. Morawetz, Bodenradarmessung im antiken Stadtgebiet von Flavia Solva, AÖ 11/2, 2000, $45 \mathrm{f}$.

23 St. Groh, Bauformen im Kastellvicus von Mautern, in: ders. (Hrsg.), Die Grabung 1998 im Kastellvicus Süd von Mautern an der Donau/Favianis, 1. ErghÖJh (2001) 36 ff. Abb. 39.
} 


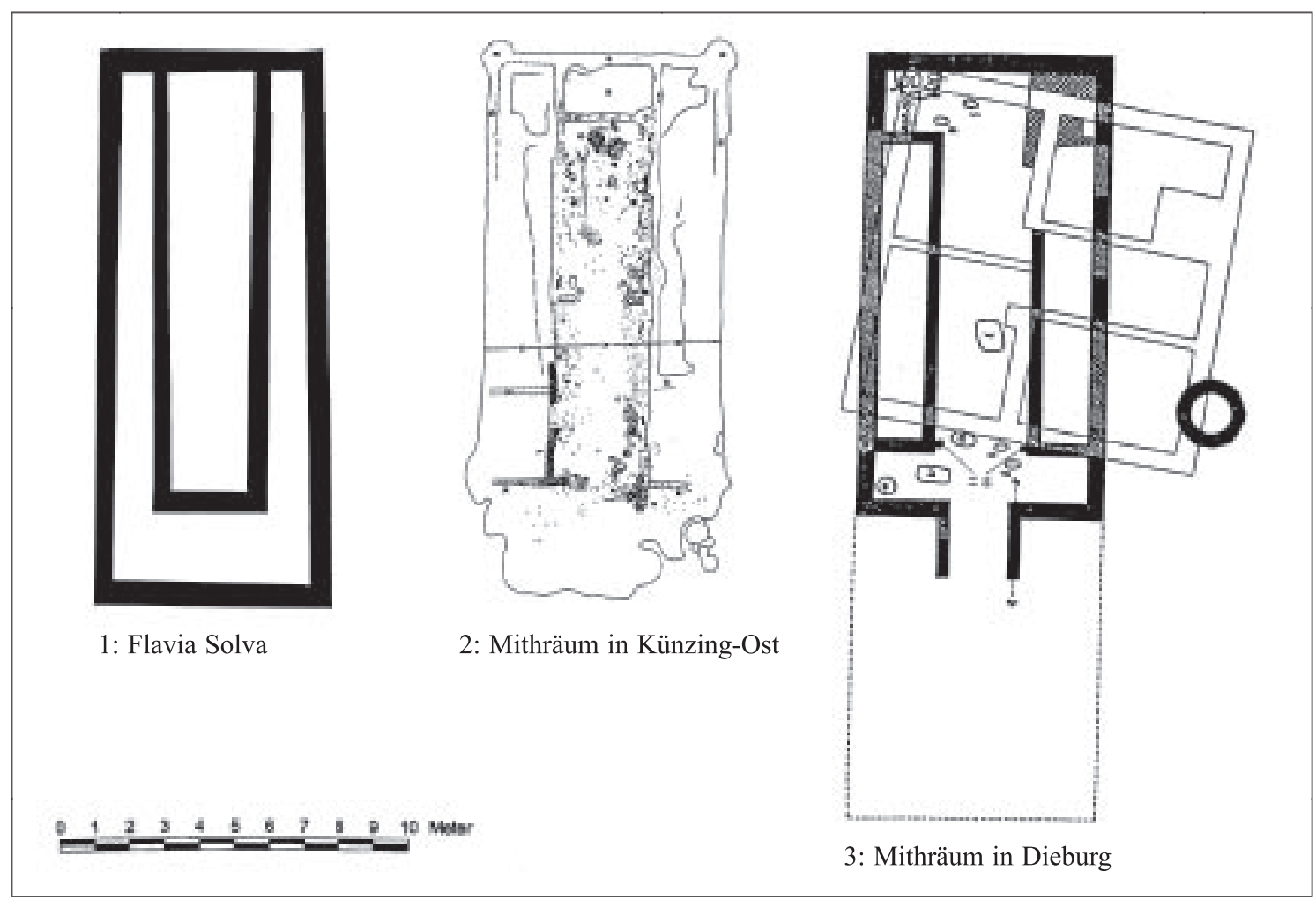

8 Langgestreckter Bau in der Insula XXIII von Flavia Solva

gebotenen Vorsicht, an die Grundrisse von Mithräen, die sich aus einem Vorraum, einem durchschnittlich 2-3 m breiten Mittelgang und den beiden 1-1,5 m breiten Podien oder Praesepia zusammensetzten $^{24}$.

Den westlichsten Teil dieser Parzelle nahm ein leicht schräg zum Straßenraster verlaufendes, isoliert stehendes Gebäude ein, dessen Mauern jedoch nur unvollständig erhalten geblieben bzw. erkennbar sind. Hierbei könnte es sich um eine jüngere Bebauung handeln, die bereits, vergleichbar mit mehreren hallenartigen Gebäuden am Nordrand der Stadt (Insulae XXVII-Nord und XXVIII), nicht mehr nach dem orthogonalen Straßenraster ausgerichtet war.

Im südöstlichen Viertel der Insula war ein großes Wohnhaus mit $23 \times 34$ m Grundfläche in den tieferen Amplitudenflächenplänen klar zu erkennen. Das Gebäude nahm eine Gesamtfläche von mindestens $780 \mathrm{~m}^{2}$ ein (Abb. 9) und entspricht in seiner Grundrißgestaltung einem rechteckigen Peristylhaus, vergleichbar mit den Peristylhäusern in der Insula V. Der Innenhof 15 dürfte ursprünglich $22 \times 12 \mathrm{~m}$ gemessen haben, die den Hof flankiernde Portikus war ca. $2 \mathrm{~m}$ breit. Der Zugang könnte am ehesten von Osten, der Straße E aus erfolgt sein, wo am Rande des Prospektionsareals ein mittig gelegener, $1,6 \mathrm{~m}$ breiter Korridor 13 bestanden hat. Die beiden quadratischen Räume $1(4,5 \times 4,5 \mathrm{~m}), 11(4 \times 4 \mathrm{~m})$ und der Korridor 13 waren in 1,8 m Tiefe entweder hypokaustiert oder mit einem Mörtelboden versehen; in 1,2 m Tiefe scheinen vor allem die südlichen Räume 3-8 und Teile der Portikus mit qualitätvollen Böden versehen gewesen

\footnotetext{
${ }^{24}$ A. Hensen, Tempel des Mithras in Südwestdeutschland, in: K. Schmotz (Hrsg.), Vorträge des 18. Niederbayerischen Archäologentages (2000) 93 ff. bzw. K. Schmotz, Der Mithrastempel von Künzing, Lkr. Deggendorf, in: Schmotz a. O. $111 \mathrm{ff}$. Bislang liegen aus dem ca. 6 km nördlich von Flavia Solva gelegenen St. Veit am Vogau ein Fragment eines mithrischen Denkmals und aus der Stadtrandinsula XLI ein Schlangengefäß (2. Hälfte des 2. Jhs. n. Chr.) vor, das vielleicht als Indiz für ein Mithräum im Stadtgebiet gehalten werden könnte (St. Groh, Ein römisches Schlangengefäß aus Flavia Solva, SchSt 17, 1994, 87 ff.).
} 
zu sein. Im Gegensatz zu dem Peristylhaus in der benachbarten Insula XXVI, das in seinem Grundriß während der gesamten Nutzungszeit kaum massive Ein- und Umbauten erfahren hatte, nahm man in diesem Haus zahlreiche Änderungen vor. $\mathrm{Ob}$ das mit einem Funktionswechsel im $\mathrm{Zu}$ sammenhang $\mathrm{zu}$ sehen ist, bleibt dahingestellt, wäre doch im privaten Bereich im Zuge oftmaliger Besitzerwechsel mit zahlreichen Umbauten und Neuparzellierungen $\mathrm{zu}$ rechnen. Im Norden dürfte dem Gebäude ein ungefähr $35 \times 15 \mathrm{~m}$ großer Hof oder Garten vorgelagert gewesen sein, der in einer jüngeren Phase

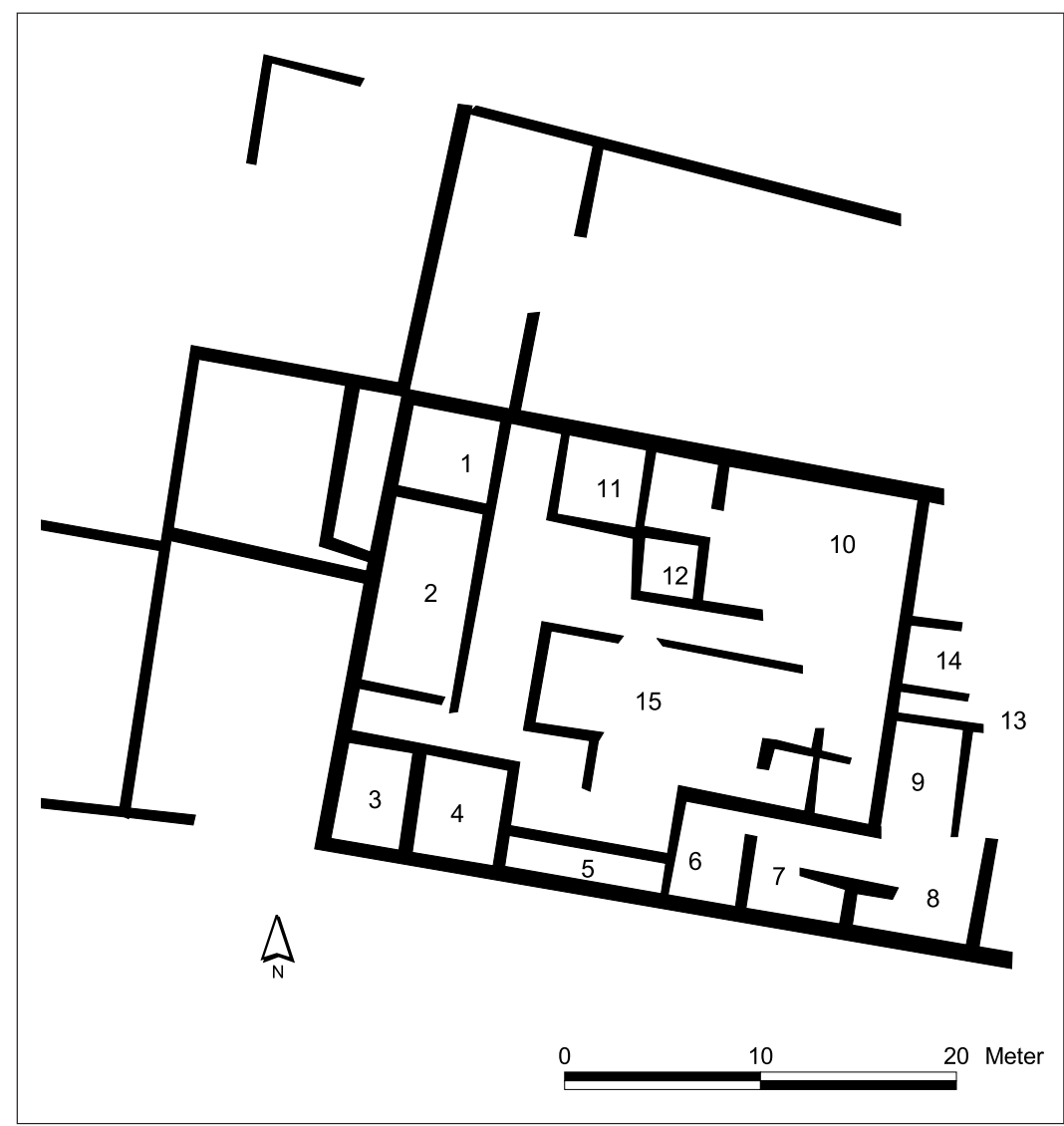

9 Flavia Solva. Peristylhaus in der Insula XXIII-Ost als eigene Parzelle abgetrennt und mit einem weiteren Wohnhaus überbaut wurde. Die seichteren Überbauungen reichen im Norden weit in den Verlauf der Straße L zum benachbarten Forum hinaus.

Aufgrund der Widerstandsmessungen 1998 wurde noch vermutet, daß es sich im Bereich dieser Insula um ein größeres monumentales Gebäude öffentlichen oder kultischen Charakters gehandelt haben könnte. Ein monumentales Gebäude in dieser Insula konnte durch die Georadarmessung aber nicht bestätigt werden.

Im Süden dürfte dieser Insula entlang der Straße K ein Laubengang vorgelagert gewesen sein. Eine kreisförmige Anomalie zwischen dem Laubengang und der Straßenaufschüttung könnte auf einen größeren, öffentlichen Brunnen hinweisen, es könnte sich aber auch um einen Bombentrichter aus dem Zweiten Weltkrieg handeln.

\section{Insula XXV}

\section{Forschungsgeschichte}

Die Insula XXV wurde 1919 von W. Schmid in seinen Gesamtplan der Stadt als Gebäudeblock unbekannter Ausdehnung an der Straße E aufgenommen (Abb. 2a) ${ }^{25}, 1979$ erfolgten Grabungen unter E. Hudeczek. In einem nördlichen 'Vorbau' der Insula konnten dabei »unter einer Ziegelund Hüttenlehmzerstörungsschicht ein großer geschlossener Komplex von Keramik des 4. Jhs. n.Chr.« geborgen werden ${ }^{26}$. Die Beschreibung des Befundes und die Stratigraphie deuten auf

\footnotetext{
25 Schmid (Anm. 19) 141 Abb. 61.

26 E. Hudeczek, Grabung Flavia Solva, JberJoanneum 1978, N. F. 8, 1979, 114.
} 

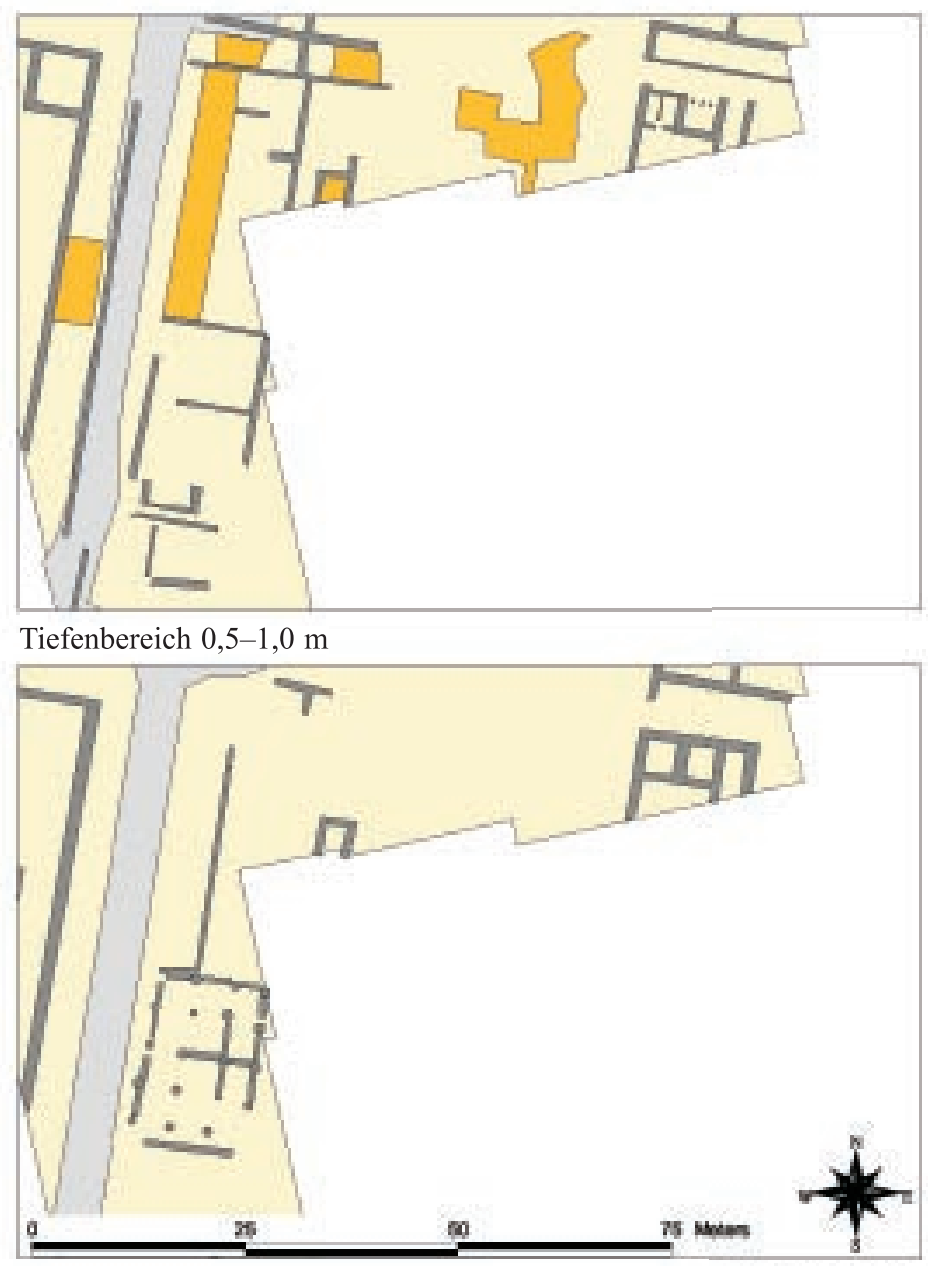

Tiefenbereich $1,0-1,5 \mathrm{~m}$

Legende

Estrich

Mauer

10 Flavia Solva. Interpretation der Georadarmessungen in der Insula XXV eine mehrphasige kaiserzeitliche Bebauung des Areals und eine spätantike Nutzung, die durch eine Zerstörung im oder nach dem 4. nachchristlichen Jahrhundert ihr Ende fand. Von besonderem Interesse ist die in den Grabungen dokumentierte Ausdehnung der vor allem im Bereich der Insula XL situierten frühesten Ansiedlung von Flavia Solva auch auf die - durch die Straße M getrennte - weiter südlich gelegene Insula XXV.

Von den Grabungen des Jahres 1979 lagen bis zum Zeitpunkt der geophysikalischen Messungen keine Pläne vor.

Interpretation der geophysikalischen Prospektionsergebnisse

Wesentliche Teile im Osten der Insula XXV liegen ihm Bereich eines modernen Wohnhauses und waren daher für eine Messung nicht zugänglich. Die Insula, die Ausmaße von mindestens $60 \times 65 \mathrm{~m}$ aufweist, wurde durch die Georadarmessung auf einer Fläche von $1350 \mathrm{~m}^{2}$ erfaßt.

Die Verbauung des untersuchten Areals gliedert sich in drei Abschnitte (Abb. 10): Der Westteil wurde bis zu einer Breite von 20 m flächig verbaut, der Mittel- und Ostteil dürften, zumindest im Norden, bis zu dem bei der Besprechung der Insula XL erwähnten Magazin, wahrscheinlich Bestandteil eines Flußhafens, frei von Verbauung gewesen sein. Die westliche Gebäudeflucht springt im Vergleich zur Außenmauer des Hauses 1 in der nördlichen Insula XL um ca. $4 \mathrm{~m}$ in den Straßenbereich vor. Entlang der Straße E ist im nördlichen Teil eine lange, $4 \mathrm{~m}$ breite Halle mit Estrichresten zu erkennen, an die nach Osten eine weitere, $8 \mathrm{~m}$ breite Halle anzuschließen scheint. Eine Dreiteilung dieser ca. $30 \times 12 \mathrm{~m}$ großen Halle ist nicht auszuschließen. Östlich dieser Halle scheint eine Reihe quadratischer Räume bestanden zu haben. Im Süden läßt sich auf einer Fläche von $18,3 \times 13,5$ m eine Säulenstellung mit $4 \times 5$ Säulen mit einem mittleren Säulenabstand von 4,3 m klar ausmachen. Die Fundamenttiefe beträgt 1,5 m, in einer Tiefe von 1,1 m scheinen die Säulen mit Mauern verbunden worden zu sein, d. h., ein gitterförmiges Fundament wurde unter bzw. zwischen den Säulen errichtet. Die Oberkanten der Hallenaußenmauern liegen bei ca. 0,5 m unter Geländeoberkante. Südlich dieser Säulen/Fundamente sind weitere Anbauten erkennbar, die bis zur südlichen Flucht der Insulae XXX-XXVI und XXV reichen, an die südlichste Säulenstellung folgte wahrscheinlich ein ca. $7 \mathrm{~m}$ breiter und 1,1 m 
tief fundamentierter, quergelagerter Korridor mit einem mittig plazierten apsidialen oder quadratischen Abschluß.

Die Straße E wird durch die baulichen Maßnahmen in der Insula XXVI und XXV bis auf eine Breite von $6 \mathrm{~m}$, das entspricht der Breite der Schotterungen ohne flankierende Gehsteige bzw. Laubengänge, reduziert. Daraus ließe sich eine geplante platzartige Ausgestaltung des Gebietes zwischen den Insulae XXVI-Ost und XXV-West ableiten. Aufgrund dieses Grundrisses und der direkten Nachbarschaft zum Forum erscheint es sehr wahrscheinlich, daß es sich bei diesem Gebäude im westlichen Teil der Insula um eine Basilika mit einem Mindestausmaß von $54 \times 18 \mathrm{~m}$ handelt (s. u.).

\section{Insula XXVI}

\section{Forschungsgeschichte}

Im südlichen und nördlichen Stadtgebiet von Flavia Solva führte W. Schmid 1911 im Auftrag des Österreichischen Archäologischen Instituts Grabungen durch. Im Bereich der Insula XXVI legte er Teile eines Hauses in dessen Südwestecke frei (Abb. 2a) ${ }^{27}$. Er untersuchte sieben Räume dieses Hauses, das er wegen eines an die westliche Außenmauer des Raumes 6 angebauten vermeintlichen Töpferofens als »Haus des Töpfers« bezeichnete. Bei dem 'Töpferofen' handelte es sich um eine im Grundriß 1,35 m lange, 0,38 m schmale und 0,36 m hohe überwölbte Kammer, in die zwei kaminartige mit runden, bandförmigen Reifen aus Eisen verstärkte und mit Lehm verkleidete Ausnehmungen eingelassen waren. Man errichtete das Objekt auf einer $2,52 \times 1,11 \mathrm{~m}$ großen, 0,04 m starken Arbeitsfläche aus gestampftem Lehm ${ }^{28}$. Eine Beschickung des Ofens erfolgte von der Nordseite. Sowohl der Grundriß des Ofens als auch dessen funktionaler Aufbau schließen eine Interpretation als Töpferofen aus ${ }^{29}$, vielmehr könnte es sich um einen sekundär an die Außenmauer des Gebäudes angebauten Backofen oder Ofen sonstiger Brennvorgänge mit niedrigen Brenntemperaturen handeln.

Im Bereich des Hauses untersuchte W. Schmid die westliche Außenmauer über eine Länge von 30,4 m, die Südmauer über eine Länge von $20 \mathrm{~m}$ und die Inneneinteilung dieses Areals (sieben Räume) nur entlang der Mauerkronen bzw. bis in geringe Tiefe. Im Südteil des Hauses (Raum 6/7) wurden »ausgedehnte Mörtelestriche« freigelegt, in Raum 2 kann anhand eines 1,8 m tiefen Grabungsschnittes und den von W. Schmid angegebenen Tiefen ein Profil rekonstruiert werden. Dies soll den Ergebnissen der Prospektion gegenübergestellt werden:

Tabelle 1: Rekonstruktion der Stratigraphie durch Raum 2 in Haus 1 (Insula XXVI) anhand der Grabungen 1911 und der geophysikalischen Prospektion mit Georadar 2000

\begin{tabular}{|l|l|l|l|l|}
\hline Tiefe (m) & Raum 2/Schmid 1911 & Interpretation & Prospektion 2000 & Tiefe (m) \\
\hline $0-0,3$ & Rezenter Humus & Humus & Humus/Erde & $0-3$ \\
\hline $0,3-0,7$ & Erde mit Mauerbruch und Asche & Schuttlage & Schuttlage & $0,3-1$ \\
\hline $0,7-0,8$ & Erde mit Sand & Boden 1? & & \\
\hline $0,8-0,82$ & Schwarze Erdschicht & Aufplanierung & & \\
\hline $0,82-1,05$ & Mauerbruch & $\begin{array}{l}\text { Schuttlage über } \\
\text { Boden }\end{array}$ & & \\
\hline $1,05-1,06$ & $\begin{array}{l}\text { Estrich aus Kieseln, lichtbraunem } \\
\text { Sand und wenig Ziegelmehl }\end{array}$ & Estrich, Boden 2 & Estrich & $1,0-1,1$ \\
\hline $1,06-1,62$ & $\begin{array}{l}\text { Bruchsteinunterlage aus Bruchsteinen } \\
\text { und Schotter, Lehm }\end{array}$ & $\begin{array}{l}\text { Estrichbürste, } \\
\text { Aufplanierung }\end{array}$ & $\begin{array}{l}\text { Mauerfundament- } \\
\text { Unterkante }\end{array}$ & max. 1,7 \\
\hline
\end{tabular}

\footnotetext{
27 Schmid (Anm. 19) $40 \mathrm{ff}$.

28 Schmid (Anm. 19) 45.

29 R. Risy, Römerzeitliche Brennöfen in Noricum (ungedr. Mag. Wien 1994) 87 ff.
} 

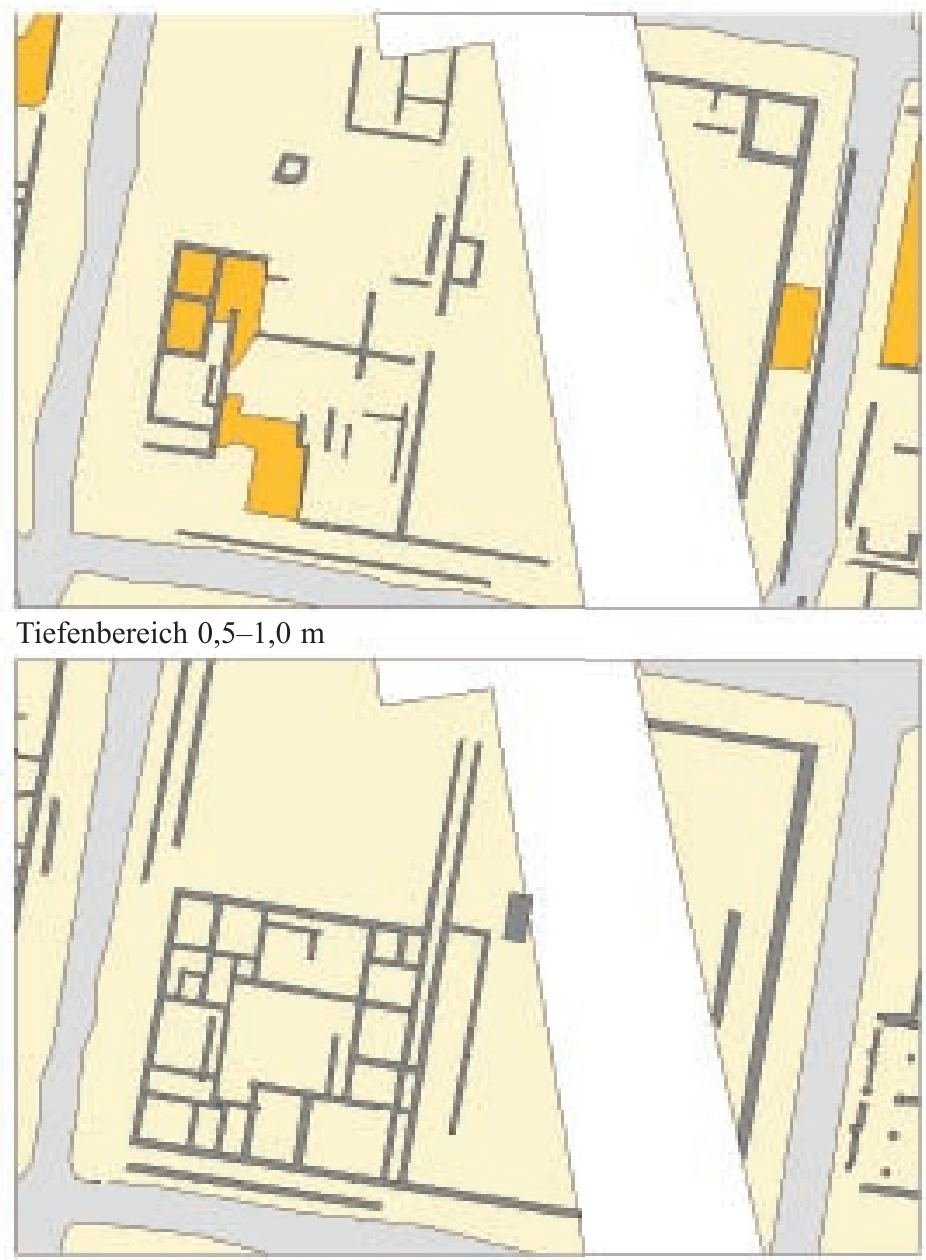

Tiefenbereich $1,0-1,5 \mathrm{~m}$

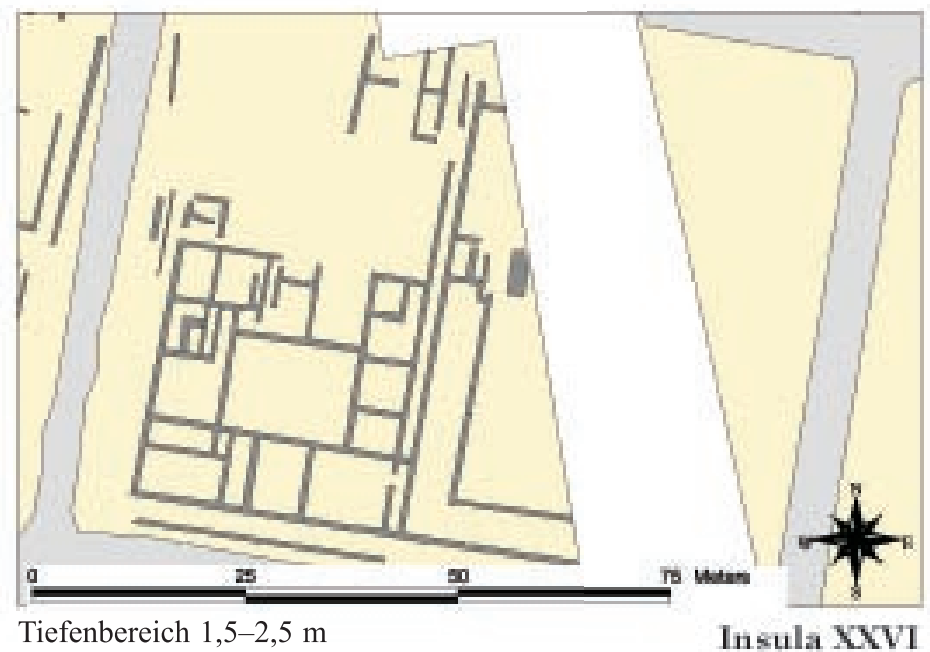

Legende

Estrich

Mauer

Straße

Prospektierte Fläche

11 Flavia Solva. Interpretation der Georadarmessungen in der Insula XXVI
Anhand der Profilbeschreibung von W. Schmid läßt sich eine mehrphasige Verbauung rekonstruieren, mit wahrscheinlich einem ersten Gehniveau (Boden 1) in ca. 0,8 m unter der Geländeoberkante und einem Estrich (Boden 2) in ca. $1 \mathrm{~m}$ unter Niveau. Die Bruchsteinlage unter dem Boden 2 deutet auf zumindest eine weitere tiefer liegende Planierung, tiefer als 1,6 m. Die Außenmauern waren $0,5-0,6 \mathrm{~m}$ stark, reichten über $1,4 \mathrm{~m}$ unter die Geländeoberkante und waren in der Südwestecke des Hauses mit einem Eckstein (Sandsteinquader) verstärkt.

Südlich des Hauses untersuchte W. Schmid die Straße L, wo in einer Tiefe von $0,5 \mathrm{~m}$ eine Schotterung dokumentiert wurde.

Nördlich des Hauses traf der Ausgräber mit Hilfe eines $4 \mathrm{~m}$ breiten Suchgrabens in $30,2 \mathrm{~m}$ Entfernung von der Nordwestecke auf eine $0,55 \mathrm{~m}$ breite und $0,27 \mathrm{~m}$ hohe, qualitativ minderwertige Bruchsteinmauer, an die im Süden eine Pflasterung aus Sandsteinen auf einer Bürste aus Kieselsteinen ansetzte. Das Fundmaterial dieser Grabungen ergab ein Keramik- und Münzspektrum des ausgehenden 1 . bis 4. Jahrhunderts n. Chr., aus den Grabungen im sog. Haus des Töpfers stammen Münzen des Traian und Constantin II, dem Suchgraben nördlich dieses Hauses können Münzen des Hadrian, Gallienus, Constantin II, Valens, Iovanis und Valentinian I zugewiesen werden.

1995-1999 fanden unter E. Hudeczek Grabungen in der Nordwestecke der Insula XXVI statt. Im Zuge dieser konnten zusammen mit Befunden in der Südostecke der Insula XXIX und Südwestecke der Insula XVII-Süd Werkstättengebäude 